J Am Med Dir Assoc. 2014 December ; 15(12): 881-884. doi:10.1016/j.jamda.2014.07.009.

\title{
Hospice in the Nursing Home: Perspectives of Front Line Nursing Home Staff
}

\author{
Kathleen T. Unroe, MD, MHA ${ }^{a, b, c}$, John G. Cagle, PhD ${ }^{d}$, M. E. Dennis, BA ${ }^{b}$, Kathleen A. \\ Lane, $\mathbf{M S}^{\mathrm{c}}$, Christopher M. Callahan, MD ${ }^{\mathrm{a}, b, c}$, and Susan Miller, $\mathbf{P h D}^{\mathrm{e}}$ \\ andiana University Center for Aging Research, Indianapolis, IN \\ ${ }^{\mathrm{b}}$ Regenstrief Institute, Inc., Indianapolis, IN \\ CIndiana University School of Medicine, Indianapolis, IN \\ dUniversity of Maryland-Baltimore School of Social Work, Baltimore, MD \\ eBrown University School of Public Health, Providence, RI
}

\begin{abstract}
Objective-Use of hospice has been associated with improved outcomes for nursing home residents and attitudes of nursing home staff towards hospice influences hospice referral. The objective of this study is to describe attitudes of certified nursing assistants (CNAs), nurses, and social workers towards hospice care in nursing homes.
\end{abstract}

Design, Setting and Participants-We conducted a survey of 1,859 staff from 52 Indiana nursing homes.

\begin{abstract}
Measurements-Study data include responses to 6 scaled questions and 3 open-ended qualitative prompts. In addition, respondents who cared for a resident on hospice in the nursing home were asked how often hospice: 1) makes their job easier; 2) is responsive when a patient has symptoms or is actively dying; 3 ) makes care coordination smooth; 4) is needed; 5) taught them something; 6) is appreciated by patients/families. Responses were dichotomized as always/often or sometimes/never.
\end{abstract}

Results-1229 surveys met criteria for inclusion. Of respondents, $48 \%$ were CNAs, $49 \%$ were nurses, and 3\% were social workers; $83 \%$ reported caring for a nursing home patient on hospice. The statement with the highest proportion of always/often rating was 'patient/family appreciate added care' (84\%); the lowest was 'hospice makes my job easier' (54\%). More social workers responded favorably regarding hospice responsiveness and coordination of care compared with CNAs ( $\mathrm{p}=.03$ and $\mathrm{p}=.05$ respectively).

Conclusion-A majority of staff responded favorably regarding hospice care in nursing homes. About $1 / 3$ of nursing home staff rated coordination of care lower than other aspects, and many

(C) 2014 American Medical Directors Association. Published by Elsevier Inc. All rights reserved.

Corresponding Author: Kathleen T. Unroe, Indiana University Center for Aging Research, 410 West $10^{\text {th }}$ Street, Suite 2000, Indianapolis, IN, 46202., kunroe@iupui.edu, Phone - 317-274-9235, Fax - 317-274-9305.

The authors report no conflicts of interest. 
qualitative comments highlighted examples of when hospice was not responsive to patient needs, representing important opportunities for improvement.

\section{Keywords}

Hospice; Nursing Home; CNAs

\section{Introduction}

In the United States, nearly a quarter of deaths occur in nursing homes ${ }^{1}$. Despite this, researchers have found gaps in end-of-life care in nursing homes, including pain and symptom management and advance care planning ${ }^{2-6}$. One strategy to improve care for dying residents is the involvement of hospice providers ${ }^{7}$. Hospice provides services which may not be available in a nursing home, such as specialized knowledge of symptom management, and spiritual support for residents and families. Evidence suggests use of hospice can improve outcomes for nursing home residents (e.g., better pain management, less rehospitalization, greater family satisfaction $)^{8}$. Higher intensity of hospice use has also been associated with improved end of life care processes in nursing homes ${ }^{9}$. Over the past few decades, there has been dramatic growth in hospice use by nursing home residents ${ }^{10}$. Despite this growth, however, hospice may still be underutilized ${ }^{11,12}$. Based on Medicare claims data from 2006, 67\% of nursing home decedents die without ever receiving nursing home hospice ${ }^{10}$. Late referrals also may limit the impact hospice has on quality of care at the end of life; in one analysis, nearly a quarter of nursing home hospice residents received hospice for a week or less.

Nursing home residents with a prognosis of six months or less can receive care under the Medicare Hospice Benefit, but when the benefit is activated the nursing home remains responsible for the resident's day-to-day care needs. Communication between hospice providers and nursing home staff regarding the resident's care is critical, especially around a decline or change in status. Relationships between nursing homes and hospices function best when there is open communication and collaboration in resident care planning ${ }^{13}$.

Attitudes towards hospice care held by nursing home staff are known to impact referrals to hospice for nursing home residents ${ }^{14}$. Nursing home staff with negative attitudes about hospice may be less receptive to hospice involvement. Many factors may influence staff perceptions of hospice, including beliefs about hospice, a sense of territorialism, or prior experience with hospice providers at work or in their personal lives. Relationships between hospice providers and nursing homes are strained when the nursing home staff feels hospice does not provide a valuable service to residents or they do not understand the role of hospice in the nursing home ${ }^{13}$.

A recent Canadian study examined attitudes of a variety of long term care workers towards end of life palliative care and death. They found that these workers had an overall positive attitude towards palliative care in long term care and most respondents felt they had a responsibility to help residents prepare for death ${ }^{15}$. The authors suggest that attitudes towards end of life care may be more related to the worker's training and education as opposed to exposure to taking care of dying residents. A recent Dutch study found that a 
majority of nursing staff, including those in nursing homes, want involvement in end of life care decisions; respondents felt that patients may prefer to discuss end of life care with nurses and that physicians relied on nurses' opinions about care of terminally ill patients ${ }^{16}$.

In the United States, end of life care in nursing homes is often provided by outside hospices. Attitudes towards hospice among nursing home staff are not well described; this study was designed to gather information about their opinions regarding hospice and palliative care. We describe findings from a survey of 1,859 clinically trained nursing home employees from 52 rural and urban mid-west nursing homes. We compared attitudes about hospice by position because social workers, nursing staff, and certified nursing assistants (CNAs) work with both nursing home residents and hospice providers in different capacities, and have varied education and training backgrounds. We present the results of staff answers to questions about hospice in the nursing home, as well as illustrative quotes from qualitative data to present a fuller picture of attitudes towards hospice.

\section{Methods}

This study was approved by an Institutional Review Board. The intent of this voluntary survey was outlined in an introductory cover letter, which stated that completion of the survey indicated consent to participate.

\section{Sample and Survey Administration}

Employees from 52 Indiana facilities, representing two nursing home chains, were asked to complete an anonymous survey about their practices, knowledge and opinions regarding palliative and hospice care in May-June of 2012. Surveys were distributed by research personnel to facility staff in the following roles: Certified Nursing Assistants (CNAs), Nurses (which included both Licensed Practical Nurses [LPNs] and Registered Nurses [RNs]), Social Workers ([SWs] which consisted of LCSWs, MSWs, or anyone designated by the facility to work in that capacity), or "Other." An overall response rate of $71 \%$ was calculated based on the total number of staff given an opportunity to complete the survey. The survey was administered on paper during regularly scheduled staff meetings and used an online survey tool.

We received 1859 surveys, 432 (23\%) of which were excluded due to missing information on job role, a key study variable. Of these incomplete surveys, $81 \%$ answered no hospice questions and $95 \%$ were missing all demographic variables. Of the completed surveys, we excluded 198 surveys (14\%) where the staff position indicated was 'Other' as this category could represent a multitude of job positions, including some not involved with direct resident care. Thus, 1229 (86\% of the completed surveys) surveys were available for analysis; of these, $17 \%$ did not indicate that they had cared for a nursing home resident on hospice and, therefore, were not prompted to answer the hospice questions.

\section{Survey Data Collection}

Study data included information from six scaled questions and three open-ended prompts, part of a larger survey on palliative care knowledge and practices. Questions were developed through conversations with nursing home staff and leadership regarding experiences with 
hospice, as well as consultation with experts in the field. Prior to survey administration, the questions were piloted with five staff members, 3 CNAs and 2 LPNs, at one nursing facility for readability and understandability. Respondents were first asked whether they had taken care of a resident on hospice in the nursing home and, if so, whether hospice: (1) makes their job easier; (2) is responsive; (3) care coordination is smooth; (4) is needed; (5) has taught them something; (6) is appreciated by patients/families. Responses were dichotomized into two categories: always/often or sometimes/never. Responses of always/often indicate a more positive attitude towards hospice. Respondents also provided demographic information including age, race and gender.

Qualitative data were collected from free text responses to the following prompts: "Describe a positive experience taking care of a dying patient;" "Describe a negative experience taking care of a dying patient;" and "Any other thoughts or concerns regarding palliative care, comfort care and hospice in nursing homes?" All survey respondents had the opportunity to answer the qualitative questions.

\section{Analysis}

Descriptive statistics were calculated for each position as well as the full sample. Multiple logistic regression models were used to compare the association between a respondent's position and the response of often/always to each of the six quantitative hospice questions. All models were adjusted for age and length of time working in nursing homes. Post hoc pairwise comparisons were conducted for statistically significant findings using Tukey's method. Observations missing either the outcome or covariates were excluded from the model on that outcome only.

Using the open-ended responses, we identified responses that referenced hospice care. We categorized these responses by the subjects of the six quantitative questions.

\section{Results}

Table 1 shows the characteristics of 1229 survey respondents in the analytic sample, including their positions: CNAs (48\%), nurses (which included 411 LPNs and 188 RNs $49 \%$ ) and SWs (3\%). Staff in all positions were predominantly female (93\%) and white (76\%). Overall, most staff had either worked in nursing homes between 6 months and 5 years, or over 10 years. Very few had worked less than 6 months, particularly SWs and nurses. Most SWs had worked 5-10 years or over 10 years. The majority of the staff was $\leq$ 50 years of age. CNAs were the youngest group, with $56 \%$ being between $18-30$ years old.

Of the total analytic sample, $83 \%(\mathrm{n}=1014)$ reported that they had cared for a nursing home resident enrolled in hospice. There were 87 (7.1\%) who did not answer the question and 128 (12.6\%) reported that they had not cared for a resident enrolled in hospice. These 128 respondents, when compared to the 1014 who had cared for a resident in hospice, were younger $(\mathrm{p}=0.02)$, had less experience in nursing homes $(\mathrm{p}<.001)$, and were more likely to be CNAs compared to nurses ( $\mathrm{p}<.001)$. There were no differences between the two groups on race $(\mathrm{p}=.27)$ or gender $(\mathrm{p}=.56)$. Table 2 shows the responses to the six questions related to hospice care. Across items, the proportions of responses rated as always/often ranged 
from $54 \%$ to $84 \%$. The statement with the greatest proportion who answered always/often was, 'Patients and their families appreciate the added care hospice provides' and the lowest proportion was in response to, 'Hospice staff make my job easier by doing some of the care of the patient.' By position, more SWs answered always/often, and for four of the six questions, the lowest ratings were by CNAs. Responses to two statements, "Hospice staff are responsive when a patient has symptoms or is actively dying" and "Coordinating care plans with hospice providers is a smooth process," differed depending on staff position. For both items, SWs had significantly more positive attitudes toward hospice than CNAs ( $\mathrm{p}=.03$ and $\mathrm{p}=.05$, respectively). For the latter question, nurses also had significantly more positive attitudes than CNAs ( $\mathrm{p}=.03$ ).

Of total respondents, 708 answered at least one of the general qualitative questions about care of dying patients and $26 \%(n=186)$ of these qualitative responses referred to hospice.

\section{Hospice staff make my job easier by doing some of the care of the patient-} Just over half of respondents (54\%) answered always/often to this statement. One respondent stated: "[hospice is] extremely helpful to the nursing staff to have that extra hand and gives us another voice in dealing with doctors and obtaining pain medication orders... also helpful with those difficult end-of-life talks with the families." Another respondent commented: "hospice care makes it easier for us as aides and nurses because they can be one-on-one with the family and give them $100 \%$ attention." Reported negative experiences included statements such as, [hospice did a] "poor job in communicating with our staff" and [hospice providers were] "not paying attention to the needs of the family, not respecting our staff at the facility."

\section{Hospice staff are responsive when a patient has symptoms or is actively} dying-Overall nearly three quarters (73\%) of nursing home staff answered always/often to the question. While one LPN stated that "[hospice] nurses...make sure [residents] are comfortable in their last few days of life," there were a number of comments highlighting times when hospice was insufficiently responsive to patient needs: "There have been many times when hospice is unavailable when the patient is actively dying...It makes the extra work of coordinating care seem like a waste of time."

Coordinating care plans with hospice providers is a smooth process-In the analytic sample, two thirds (66\%) of staff answered always/often to this statement. There were a few negative comments describing delays and difficulties that occurred during care coordination "causing conflict and undue stress," but others described a seamless synergy between the nursing home and hospice staff - "we worked together to ensure the resident was clean, his linens [were] changed, he was suctioned as needed, had excellent oral care, and was sufficiently medicated for pain and air hunger...hospice and our SNF staff comforted the resident, tended to his needs and ensured someone was present when he took his last breath."

Hospice provides needed care at the end of life for nursing home patients and families-Nearly $80 \%$ answered always/often and several qualitative responses addressed 
this topic. Staff stated that residents "get more care" and that having hospice involved creates a "win-win situation." Some staff made reference to the limits of their own abilities to spend one-on-one time with residents and appreciated that hospice staff could give extra attention to dying residents. Others suggested that hospice care in the nursing home was unnecessary or, at least, overlapped with service provided by the facility staff: "we do the same things regardless of hospice being here, we can provide the same medications and call doctors just as easily as hospice." Another LPN stated that prior to hospice availability in nursing homes "we were able to take care of patients without it." Others expressed a feeling that hospice had more value in a home setting rather than the nursing home.

\section{I have learned something new about taking care of dying patients from} hospice staff-A majority, 59\%, answered always/often. Nursing home staff commented that they had learned from hospice staff about "making the resident comfortable and calm" and that hospice staff had taught facility staff "what to expect, signs of pain...many other helpful hints that helped to give excellent patient care."

Patients and their families appreciate the added care hospice provides-This statement was answered always/often by $84 \%$ of staff. Some highlighted that hospice is a "comfort" to families and provides additional education and services. Another spoke highly of how "hospice follows up with the grieving family after death." Another social worker mentioned that a family had thanked her for referring their family member to hospice. Several staff members commented on their frustration with families who resist hospice referral or have "unrealistic" expectations for the resident near the end of life.

\section{Discussion}

We found that a majority of nursing home staff responded favorably regarding hospice services. While a physician certifies that a patient is eligible for the hospice benefit, the attitudes of front line staff can have an important influence on decision-making near the end of life given their frequent interactions with nursing home residents and families.

Quantitative and qualitative data provided by this large survey provide insight into the attitudes of nursing home staff who work at the bedsides of these frail residents. A few findings that should be highlighted include the strong perception that families and residents benefit from hospice in the nursing home, concerns about coordination of care, and the highly favorable attitudes of social workers towards hospice.

A higher proportion of nursing home staff rated statements favorably related to the benefit of hospice for residents and families - "hospice staff are responsive," "provides needed care," "patients appreciate hospice" - compared to statements related to the support of hospice to them, the staff. Comparatively fewer staff felt that hospice consistently made their jobs easier, that they had learned something from hospice providers, or that coordinating care plans was a smooth process. About $1 / 3$ felt that smooth coordination of care plans occurred only "sometimes" or "never" and most of the related qualitative comments were negative. This may represent an important opportunity for improvement in communication between hospice providers and nursing homes. 
Across all quantitative survey questions, social workers had consistently better impressions about hospice compared to their nursing trained coworkers. Social workers are often tasked with initiating family conversations about resident goals of care and, thus, they frequently refer residents and families to hospice and may be more attuned to preferences for such care. Social workers may be less knowledgeable about gaps in interagency coordination, management of physical symptoms, and hospice responsiveness. Further, social workers may be more skilled and experienced in interdisciplinary coordination. Our findings suggest that hospices may need to do a better job of educating nursing staff about their scope and limitations -- and that social workers may be natural allies for hospice and good point people for building interagency relationships and fostering a more collaborative approach to end-oflife care.

We also found other studies have reported negative comments made by nursing home staff towards hospice ${ }^{17}$. While we also report negative comments, due to our mixed methods approach we are able to place these comments in perspective. For example, there were several negative comments related to responsiveness of hospice; however, overall nearly $3 / 4$ of respondents felt that hospice was always or often responsive. Those who wrote about negative interactions may have felt very strongly about those incidents, but the overall impressions were still largely favorable towards hospice.

Limitations to this study included the survey format, which prevented us from asking follow-up questions as could be done in an interview or focus group design. While large and inclusive of both rural and urban nursing homes, this is a one state sample which limits generalizability. Further, a lack of gender or ethnic diversity limits generalizability of results. According to a state by state analysis of hospice use by nursing home residents, $33 \%$ of Indiana nursing home decedents received hospice care in 2006, which was the same as the national average of $33 \%$.

\section{Conclusion}

In this study, CNAs, nursing staff and social workers generally responded favorably in multiple domains related to the use of hospice in nursing homes. The variety of qualitative statements made by staff regarding hospice provide insight into everyday experiences, both positive and negative, when caring for dying residents in nursing homes, as well as highlight areas for improvement, such as in coordination of care. The attitudes of front-line nursing home staff who interact on a daily basis with residents and their families, are important to understand as hospices, nursing homes and medical providers seek to improve quality of end-of-life care in nursing homes and optimize appropriate and timely referral to hospice.

\section{Acknowledgments}

Supported by NPCRC grant \#4183655, NIA grant RO1 AG031222 and K24 AG024078. All authors were involved in the analysis, writing and editing of this manuscript. 


\section{References}

1. Mitchell SL, Teno JM, Miller SC, Mor V. A national study of the location of death for older persons with dementia. Journal of the American Geriatrics Society. Feb; 2005 53(2):299-305. [PubMed: 15673356]

2. Cohen-Mansfield J, Lipson S. Pain in cognitively impaired nursing home residents: how well are physicians diagnosing it? Journal of the American Geriatrics Society. Jun; 2002 50(6):1039-1044. [PubMed: 12110063]

3. Hanson LC, Eckert JK, Dobbs D, et al. Symptom experience of dying long-term care residents. Journal of the American Geriatrics Society. Jan; 2008 56(1):91-98. [PubMed: 17727647]

4. Miller SC, Mor V, Teno J. Hospice enrollment and pain assessment and management in nursing homes. Journal of pain and symptom management. Sep; 2003 26(3):791-799. [PubMed: 12967728]

5. Mitchell SL, Teno JM, Kiely DK, et al. The clinical course of advanced dementia. The New England journal of medicine. Oct 15; 2009 361(16):1529-1538. [PubMed: 19828530]

6. Teno JM, Clarridge BR, Casey V, et al. Family perspectives on end-of-life care at the last place of care. JAMA : the journal of the American Medical Association. Jan 7; 2004 291(1):88-93.

7. Meier DE, Lim B, Carlson MD. Raising the standard: palliative care in nursing homes. Health Aff (Millwood). Jan-Feb;2010 29(1):136-140. [PubMed: 20048372]

8. Lorenz K, Lynn J, Morton SC, et al. End-of-Life Care and Outcomes. Vol Evidence Report/ Technology Assessment 110: Agency for Healthcare Research and Quality. 2004

9. Zheng NT, Temkin-Greener H. End-of-life care in nursing homes: the importance of CNA staff communication. Journal of the American Medical Directors Association. Sep; 2010 11(7):494-499. [PubMed: 20816337]

10. Miller SC, Lima J, Gozalo PL, Mor V. The growth of hospice care in U.S. nursing homes. Journal of the American Geriatrics Society. Aug; 2010 58(8):1481-1488. [PubMed: 20646101]

11. Stevenson DG, Bramson JS. Hospice care in the nursing home setting: a review of the literature. Journal of pain and symptom management. Sep; 2009 38(3):440-451. [PubMed: 19735904]

12. Han B, Remsburg RE, McAuley WJ, Keay TJ, Travis SS. National trends in adult hospice use: 1991-1992 to 1999-2000. Health affairs (Project Hope). May-Jun;2006 25(3):792-799. [PubMed: 16684745]

13. Miller, SC. A Model for Collaborative Success-Through Collaborative Solutions. 2013. 2007 http://www.nhpco.org/sites/default/files/public/nhhp-final-report.pdf

14. Welch LC, Miller SC, Martin EW, Nanda A. Referral and timing of referral to hospice care in nursing homes: the significant role of staff members. The Gerontologist. Aug; 2008 48(4):477484. [PubMed: 18728297]

15. Leclerc BS, Lessard S, Bechennec C, Le Gal E, Benoit S, Bellerose L. Attitudes toward death, dying, end-of-life palliative care, and interdisciplinary practice in long term care workers. Journal of the American Medical Directors Association. Mar; 2014 15(3):207-213. [PubMed: 24461725]

16. Albers G, Francke AL, de Veer AJ, Bilsen J, Onwuteaka-Philipsen BD. Attitudes of nursing staff towards involvement in medical end-of-life decisions: a national survey study. Patient Educ Couns. Jan; 2014 94(1):4-9. [PubMed: 24268920]

17. Parker-Oliver D, Bickel D. Nursing home experience with hospice. Journal of the American Medical Directors Association. Mar-Apr;2002 3(2):46-50. [PubMed: 12807538] 


\section{Highlights}

- Nursing home employees completed surveys capturing attitudes toward hospice.

- The majority of staff responded favorably regarding hospice in nursing homes.

- Overall, staff felt that patients and families appreciate the added care of hospice.

- Some staff expressed concerns about coordinating care with hospice teams.

- Attitudes about hospice differed for social workers, nurses, and nursing assistants. 


\begin{tabular}{|c|c|c|c|c|c|c|}
\hline สิ & 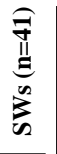 & 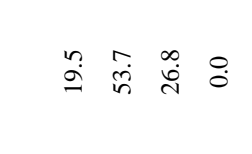 & 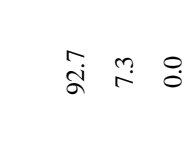 & 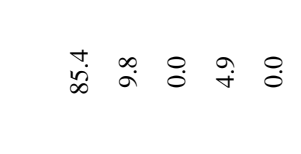 & 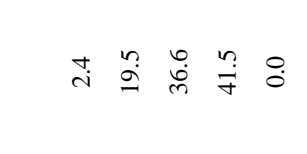 & $\stackrel{i}{i}$ ì \\
\hline 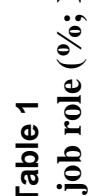 & 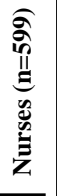 & 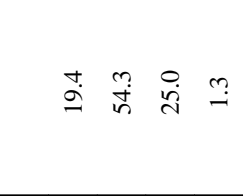 & 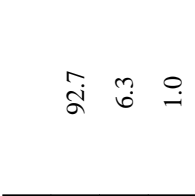 & 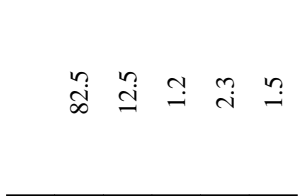 & 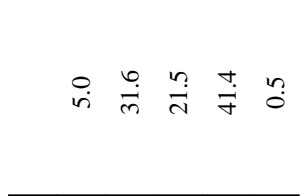 & $\stackrel{\text { ने }}{\text { के }}$ \\
\hline 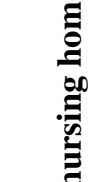 & 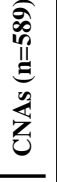 & 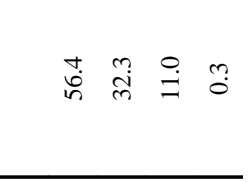 & 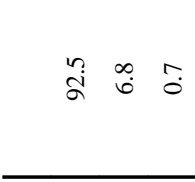 & 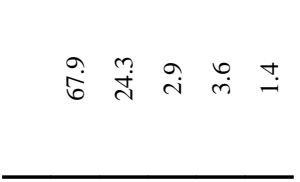 & ज̈ & $\stackrel{i}{\stackrel{i}{ \pm}} \stackrel{+}{=}$ \\
\hline 苞 & 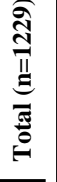 & $\overrightarrow{\vec{m}} \dot{\vec{q}} \stackrel{+}{\infty} \stackrel{\infty}{\infty}$ & $\stackrel{0}{i} \hat{b}^{\circ} \stackrel{\infty}{8}$ & 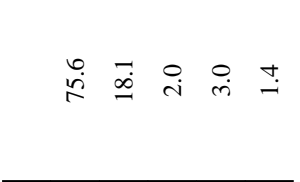 & 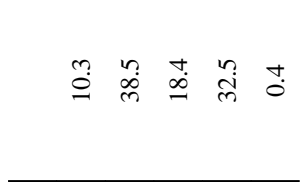 & 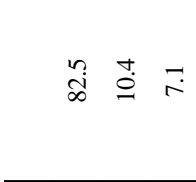 \\
\hline 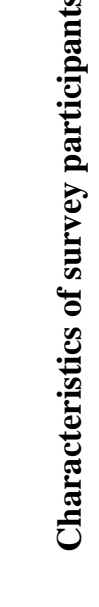 & 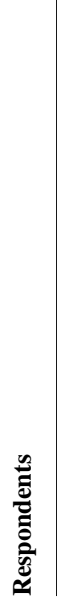 & 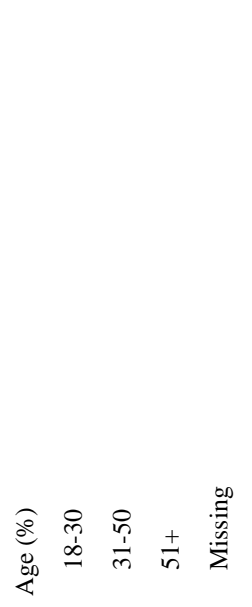 & 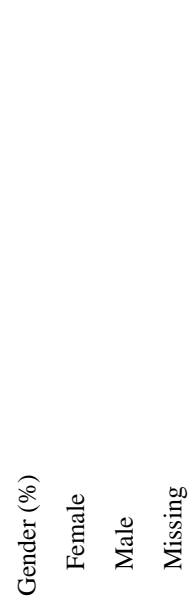 & 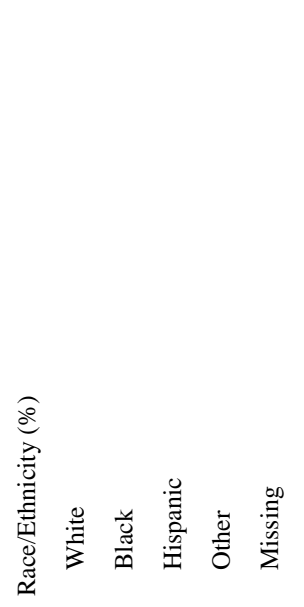 & 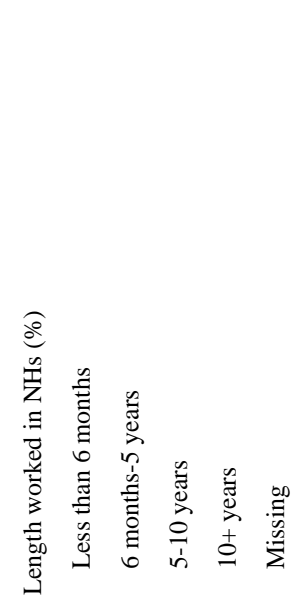 & 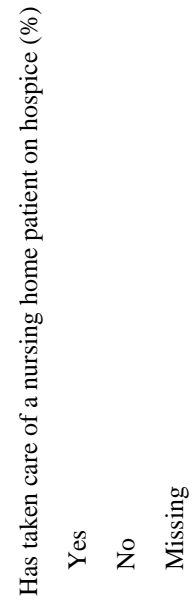 \\
\hline
\end{tabular}




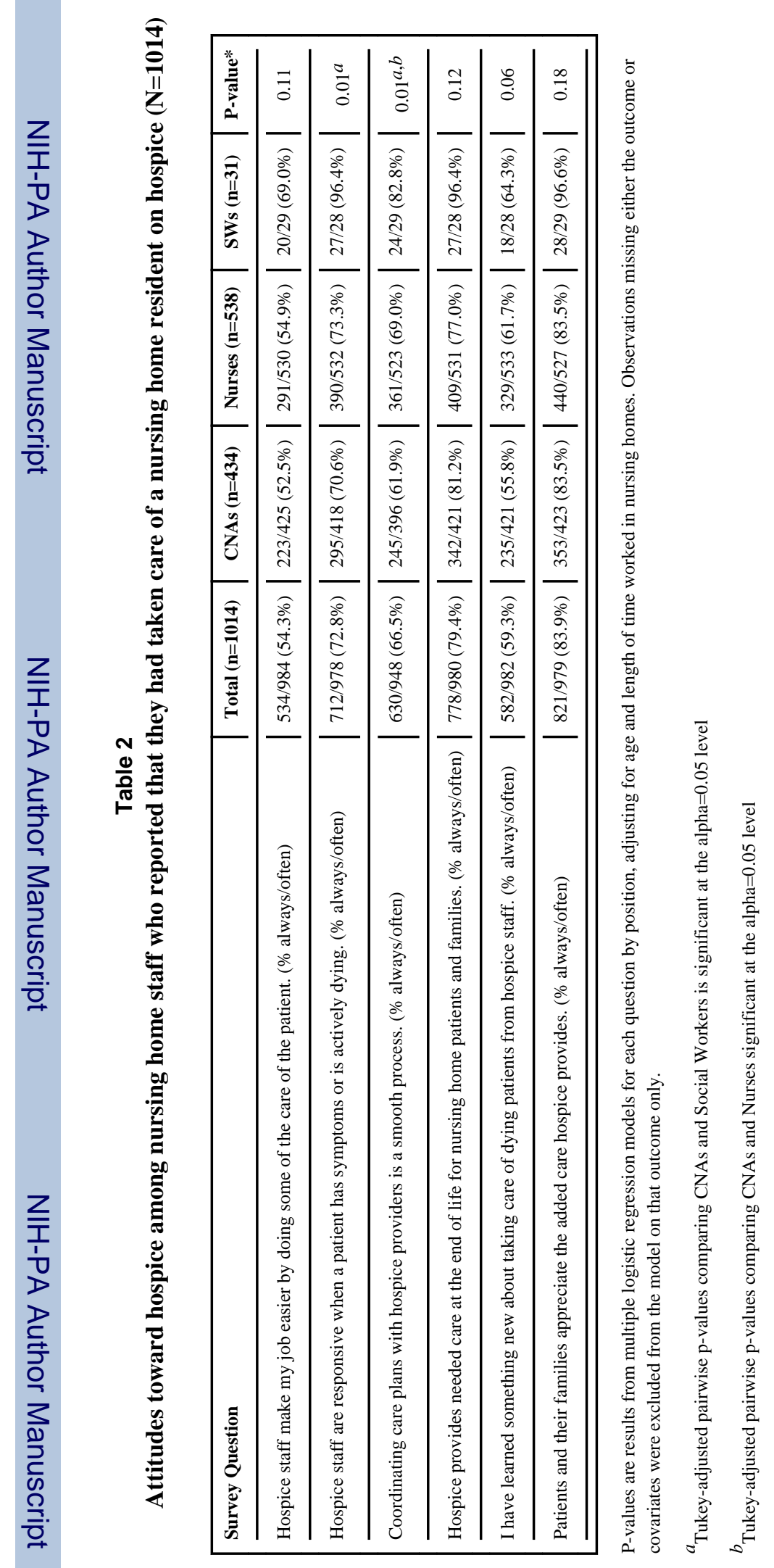

J Am Med Dir Assoc. Author manuscript; available in PMC 2015 December 01. 